\title{
SAÚDE E SUA FORMA DE VIVER: \\ PREVENÇÃO E CONTROLE DA HIPERTENSĀO ARTERIAL
}

\author{
Iraci dos Santos * \\ Araci Carmen Clos*
}

\begin{abstract}
RESUMO - A fim de orientar grupos da Comunidade Vassourense, inclusive, funcionários da Fundação Educacional Severino Sombra-FUSVE, quanto à necessidade de prevenir as doenças cárdiovasculares foi implementado o Projeto de Pesquisa e Extensão - SAÚDE E SUA FORMA DE VIVER. Utilizou-se a técnica de investigação mediante a Consulta de Enfermagem, estratégia utilizada para a coleta de dadose Palestras de orientação em grupo. O Projeto foi elaborado e desenvolvido por professores e alunos da Escola Superior de Enfermagem e Obstetrícia de Vassouras - Rio de Janeiro, especificadamente das Disciplinas de Enfermagem Fundamental e Administração Aplicada à Enfermagem, no ano-de 1991.
\end{abstract}

\begin{abstract}
The project of research and extension - Health and your way of living - have been set up in order to orient groups from Vassouras Community including employers of "Fundação Educacional Severino Sombra - FUSVE". Its main rule is the prevention from heart diseases. It has been the clinical investigation techniques since the Nursing Deliberation, a strategy to gather datum and group orientation lectures the project has been elaborated and developed by professors and students from "Escola Superior de Enfermagem e Obstetrícia de Vassouras - Rio de Janeiro", and specifically from the subjects of Basic Nursing and Management in Nursing, in 1991.
\end{abstract}

\section{INTRODUÇĀO}

As doenças cardiovasculares são responsáveis pela maior incidência de mortalidade no Brasil, e entre os fatores de risco dessas patologias destacam-se a hipertensão arterial e a obesidade.

Isto posto, a hipertensão arterial é um problema de saude pública e como tal deu origem ao Programa Nacional de Educação e Controle dá Hipertensâo Arterial (PNECHA) desenvolvido pelo Ministério da Saúde.

A gravidade co problema hipertensão arterial e a simplicidade do seu diagnóstico e de sua prevenção expressam um desafio aos profissionais da saude, exigindo dos mesmos intervenções em niveis de prevenção primária e prevenção secundária.

Assim, o Departamento de Enfermagem Fundamental da Escola Superior de Enfermagem e Obstetricia da FUSVE - Vassouras R.J. resolveu desenvolver este Projeto para contribuir com a prevenção das doenças cardio- vasculares na comunidade de Vassouras, envolvendo docentes enfermeiros e estudantes de enfermagem do 3, 50 e 60 perfodos académicos.

Tal Projeto tem os objetivos que se seguem:

\subsection{Geral}

- Orientar grupos da comunidade Vassourense quanto à necessidade de controle de peso e pressão arterial visando à prevenção das doenças cardiovasculares.

\subsection{Especificos}

- Introduzir e implementar a Consulta de Enfermagem para grupos da Comunidade de Vassouras.

- Produzir o livro "SAÚDE E SUA FORMA DE VIVER" para orientaçăo de clientes ná prevenção da hipertensảo arterial e doenças cardiovasculares.

- Publicar e divulgar o livro "SAÚDE E

\footnotetext{
* Professoras Titulares da Escola Superior de Enfermageme Obstetrl cia da Furdageo Educacional Severino Sombra. Vasuouves, Rio de Janeiro.
} 
SUA FORMA DE VIVER" junto às Instituições de Saúde e de Ensino Superior em nível nacional.

- Divulgar orientações sobre saúde através da Consulta de Enfermagem distribuindo exemplares da publicação "SAÚDE E SUA FORMA DE VIVER".

- Agendar três consultas de Enfermagem consecutivas para grupos da comunidade visando à educação para a saúde, aferiçñ̄o de peso, estatura, pulso e pressão arterial.

- Implantar o Programa de Prevenção da Hipertensão Arterial junto aos funcionários da Fundação Educacional Severino Sombra | FUSVE.

- Proceder ao diagnóstico da situação de saúde referente à prevenção e controle das doenças cardiovasculares de grupos da comunidade a partir da aferição da pressão arterial segundo a classsificação do Ministério da Saúde prevista no PNECHA.

\section{REFERENCIAL TEO̊RICO}

Para fundamentar o desenvolvimento deste projeto foram utilizadas as Normas Técnicas para o Programa Nacional de Educação e Controle da Hipertensāo Arterial (PNECHA) editada pelo MINISTÉRIO DA SAÚDE, SECRETARIA NACIONAL DE PROGRAMAS ESPECIAIS DE SAÚDE.

Visando ao maior entendimento da Comunidade, principalmente, das pessoas de nível primário de escolaridade e/ou leigas em assuntos de saúde, foi elaborado pelas autoras um Livreto, intitulado "SAÚDE - SUA FORMA DE VIVER", destinado à doação aos clientes durante às Consultas de Enfermagem e Palestras de orientação em grupo.

Tal obra foi editada e ilustrada em linguagem e forma simples e acessível à referida Comunidade conforme se descreve em seguida.

O CORPO é um dos bens que a pessoa realmente possui. Para usufruir das vantagens (andar, ouvir, cantar, brincar, trabalhar, dançar, sentir, correr e outras), que ele oferece, é necessário ter SAÚDE, pois esta é o combustíve, a energia, que faz o corpo viver. É a saúde que faz o corpo "viver bem" e é o que faz o corpo ter saude.

Portanto, a maneira da pessoa viver pode conduzir à saúde e/ou à doença. O VIVER, entretanto é algo tão natural que a gente só percebe que VIVE quando se sente: "pesado", "cansado", com "dor de cabeça", "tonteira" "perturbação visual", "pulso batendo rápido" quando apressa o passo; a gente quer ler e as "letras se embaralham".

Af, finalmente, a gente percebe que está "ansiosa" e "deprimida" e, então, já está com $100 \mathrm{~kg}$ de Peso e 14 x 10 de Pressāo Arterial.
VIVER BEM e algo que, tamberm, pode ser natural ao homem, apesar de dificuldades tais como: baixos salários, moradia e pouca alimentação; depende de sua própria decisão.

Vejamos: O ANDAR faz parte do VIVER $B E M$, não depende de dinheiro e pode ser feito até dentro de casa e/ou no trabalho - SUBINDO ESCADAS, por exemplo. Evite utilizar ônibus ou carro quando puder andar a pé.

Por isso, "MEXA-SE, SAÚDE É O QUE INTERESSA" pois contribui para a normalidade da CIRCULAÇÃO do seu SANGUE nas veias e artérias, levando a oxigenação adequada de seus: CÉREBRO, CORAÇĀO, PELE, NERVOS, MÚSCULOS, OSSOS e outros tecidos corporais, MEXA-SE com freqüência. Se puder fazer EXERCÍCIOS FISICOS, faça-os de 3 a 5 vezes por semana. Se resolver CAMINHAR, faça-o com passo rápido, diariamente e, sem parar, pelo menos, 30 minutos.

Quanto à duraçāo - os exercícios físicos, nadar, caminhar e andar de bicicleta devem ser feitos 110 mínimo de 20 a 30 minutos.

Quanto à intensidade - deve-se fazer exercícios sem parar e de tal forma que ao final a pessoa fique com a respiração acelerada e uma sensação de discreto cansaço.

Entretanto, para melhorar sua SAUUDE evite o excesso de exercícios.

Evite a VIDA SEDENTÁRIA (ficar parado, sentado por várias horas), ela é um fator de risco que conduz à HIPERTENSÃO ARTERIAL, ou seja, à PRESSÃO ALTA.

Ficar "parado", pode lhe deixar, tambem, mais "pesado", menos disponivel para andar, correr, brincar e gozar a VIDA, MEXA-SE!!! As atividades físicas quando feitas regularmente, auxiliam a perda de peso na pessoa obesa e reduzem, também, a pressão arterial.

Sobre a pressão arterial (P.A.), lembramos que esta deve ser controlada. A P.A. representa a força que o sangue, ao circular pelo corpo, exerce nas paredes das artérias. $O$ controle da Pressão Arterial evita as complicações decorrentes da hipertensão, tais como: insuficiência cardraca, doenças renais. Cuidado, portanto, com o aumento da P.A., ou seja, com a Pressão Alta.

A Pressão Arterial aumenta com o avanço da idade. A população adulta, com 20 anos ou mais, é a que apresenta maior freqüência de Pressão Alta devido aos fatores seguintes: hábitos alimentares irregulares (sal e gorduras em excesso); Obesidade (excesso de gordura); Tabagismo (uso de fumo), e frequentar ambientes fechados onde se fuma; Pessoas na famnlia com hipertensăo arterial, diabetes e outras doenças do aparelho circulatório. Uso de bebidas alcoбlicas; Estresse (tensōes) pela dificuldade de relacionamento no trabalho e na famnia e pelas condições de vida; Vida se- 
centária e "Diabetes Mellitus".

Para "viver bem", fique atento, vigilante ao seu PESO - produto da gravidade sobre a massa corporal. Procure observar se o seu PESO é proporcional à sua ESTATURA - distância vertical do corpo a partir dos pés até a parte mais alta da cabeça.

COMO EVITAR A OBESIDADE? Comendo o suficiente para alimentar o corpo, isto é, dar ao corpo: proténas, gorduras, sais minerais e vitaminas necessárias ao desgaste diário no trabalho, estudo e lazer.

Doces, açúcar, massas e sal em excesso, só contribuem para aumentar as gorduras do corpo pois o excesso pode não ser eliminado no desgaste normal diário;

- Evitando alimentos de conserva, molhos e temperos industrializados, pois são ricos em sal;

- Não colocando mais sal em alimentos já temperados;

- Não utilizando adoçantes artificiais freqüentemente, os quais têm sódio;

- Comendo menor quantidade de alimentos exceto, hortaliças e frutas;

- Evitando "beliscar" entre as refeições;

- Deixando de tomar bebidas alcoólicas e refrigerantes;

- Comendo, diariamente, pequenas quantidades de carnes magras;

- Comendo à vontade e diariamente verduras e legumes (de preferência crus) e frutas;

- Evitando frituras, leite gordo e derivados e diminuindo o consumo de ovos.

Enfim, CONTROLE seu PESO. Observe se as suas roupas estão ficando apertadas no seu corpo ou se pese com regularidade.

Lembre-se, o COMER é algo que nos dá muito prazer, mas este prazer pode nos causar arrependimentos quando surgem a OBESIDADE, DIABETES, DOENÇAS CARDIACAS que nos causam tantas dificuldades.

Portanto EVITE os excessos de GORDURA ANIMAL, SAL, MASSAS e DOCES.

$\mathrm{Na}$ tentativa de lhe ajudar a tomar esta decisão nós lhe indicamos a composição de sódio (substância que você deve evitar para não ficar obeso e hipertenso), de alguns alimentos.

1. Alimentos POBRES em sódio $(2 \mathrm{mEq} / 100 \mathrm{~g})$ :

- vegetais frescos - todos, exceto aipo, agrião, espinafre, cenoura, chicorea, beterraba, bróculis e acelga.

- frutas frescas - exceto lima e banana.

- cereais e derivados - arroz, farinha, macarråo e pão, exceto os que contém sal.

- gorduras, 6 leos, azeites, manteiga e margarina sem sal.

- bebidas - sucos naturais de frutas, chá, café e mate.

Tendo em vista a Educação à Saúde serão implementadas Palestras, promovendo-se assim, a orientaçao em grupos did cullunnuave, ниеdo o debate e a interação entre os clientes e os expositores.'

(Ver Cronograma de Atividades no Anexo 2).

Serão inves̀tigadas as seguintes variáveis dependentes:

- demográficas: sexo e idade; sócio-econômicas: escolaridade, profissão/atividade e local de trabalho/atividade.

- anamnese dirigida para os controles da PA, Peso, Estatura e Pulso, hábitos alimentares, uso de tabaco e bebidas alcólicas, problemas de saúde, uso de medicamentos, condições de atividades físicas e referências ao "stress".

- verificação da pressão arterial e pulso

- aferições antropométricas: peso e estatura

- outros dados de interesse clínico

- prescrição de enfermagem

- agendamento de outras consultas e observaçōes

- classificação da PA segundo o PNECHA

- encaminhamento quando necessário

As variáveis independentes compreenderão:

- Grupo Etário: adolescentes (de 13 a menos de 20 anos)

adultos (de 20 a 59 anos) e idosos (com 60 anos e mais)

- Classificação quanto ao Peso x Estatura x sexo:

Peso Normal, Baixo Peso e Obeso.

\section{Alimentos com moderado teor de sódin} (ate $2 \mathrm{mEq} / 100 \mathrm{~g}$ ):

- Leite, creme de leite, ovos, carnes frescas, de boi, aves, peixes e frutos do mar

(exceto caranguejo, mexilhão e ostras)

3. Alimentos com ALTO teor de sódio (6 a $40 \mathrm{mEq} / 100 \mathrm{~g})$

- pão francês, pão de milho, pão doce, pão integral, feijão preto, manteiga e margarina

\section{Alimentos com AL'TISSIMO teor de sódio} (mais de $40 \mathrm{mEq} / 100 \mathrm{~g}$ )

- carnes, pescados defumados e salgados, enlatados e embutidos (salsicha, lingüiça, choriço, salame), bacon, toucinho, caldo e extrato de carne, picles, azeitonas e molhos industrializados.

Vale a pena recordar, ainda, que os antiácidos e laxantes possuem alto teor de sódio e só devem ser utilizados com prescrição médica.

Alimente-se corretamente. Lembre-se, alimentar-se bem não é comer grande quantidade de alimentos. $O$ excesso de sal, açúcar e gordura pode não ser eliminado pelo corpo, ficando então, amazenado e produzindo doenças que não lhe permitem VIVER BEM. 


\section{METODOLOGIA}

A fim de realizar o referido diagnóstico de sarde da Comunidade Vassourense - R.J. será utilizado o método descritivo médiante a técnica de investigação clínica.

Serão realizadas Consultas de Enfermagem, estratégia de coleta de dados para a investigação clínica. A Consulta de Enfermagem promoverá a assistência individual através das açōes; anamnese, exame clínico, educação à saúde, evolução do cliente e encamizhamento à especialistas da área da saude, quando necessário. (Ver recursos no item 4)

\subsection{Instrumentação}

Foram elaborados Instrumentos de Coleta de Dados (Ver Anexo 1) para registro das atividades da Consulta de Enfermagem. Os referidos impressos foram submetidos à testagem visando sua validação durante a Comemoração da Semana da Enfermagem de 1991 realizada pela Escola Superior de Enfermagem e Obstetrícia de Vassouras - ESEO, FUSVE.

Após a testagem foram inclurdos no Instrumento da Coleta de Dados: Evolução do controle do PA, pulso E peso nas tres consultas subseqüentes e classificação da P.A.

\section{RECURSOS}

Os recursos humanos compreendem cinco professores das Disciplinas Enfermagem Fundamental e Administra ão Aplicada à Enferma- gem I e II e os Graduandos de Enfermagem que cursam as referidas disciplinas.

Constam dos recursos ambientais o Laba ratório de Enfermagem da ESEO, Consultório no Ambulatório do Hospital - Escola Jarbas Passaninho da FUSVE e Salas de Aulas e/ou de Conferências dessa Instituiçảo.

Os recursos documentais incluem os impressos proprios para a Consulta de Enfermagem (instrumentos de coleta de dados), cartazes para divulgaçăo das atividades, o livro "Saude e sua Forma de Viver", de autoria dos professores responsáveis por este Projeto, que o elaboraram especialmente para serem doados aos clientes a serem atendidos e os recursos audiovisuais.

Os recursos materiais para a Consulta de Enfermagem abrangem dois tensiômetros, dois estetoscópios, uma balança antropométrica, duas mesas de escritório e quatro cadeiras.

Para as palestras e registros das atividades têm sido utilizado os seguintes recursos materiais; um retroprojetor e folhas de transparências, uma televisão e video-cassete, um projetor de "slides" e tela para projeção, um quadro de-giz e apagador, seis canetas de retroprojetor, seis lápis pretos, três canetas azuis, três canetas vermelhas, três lápis de cor, três borrachas, uma regua, uma resma de papel ofício e meia resma de papel pautado.

Os recursos financeiros para aquisição dos materiais, edição e reedição do Livro "Saúde é sua forma de viver" foram doados pela Fundação Educacional Severino Sombra.

\section{BIBLIOGRAFIA}

CAS TRO, Ieda Barreira et all. Monual de Procedinento de Enfermagem: Tecnologia apropriada para coletividade de estudo e trabalho. Rio de Janeiro: Sub-Reitoria de Ensino para Graduados e Pesquisa/SR-2/UFRJ, 1986, 75p.

MINIS TÉRIO DA SAÚDE, SECRETARIA NACIONAL DE PROGRAMAS ESPECIAIS DE SAUDE. Normas
Técnicas para o Programa Nacional de Educaçāo e Controle da Hipertensão Arterial (PNCHA). Brasilia: Centro de Documentaçāo do Ministério da Saúde, 1988, 89p.

DUGAS, Beverly M. Enfermagem Prática. 3. ed. Rio de Janeiro: Interamericana, 1978, 526p. 


\section{ANEXO 1 \\ FUNDAÇĀO EDUCACIONAL SEVERINO SOMBRA \\ ESCOLA SUPERIOR DE ENFERMAGEM E OBSTETRICIA DE VASSOURAS \\ CONSULTA DE ENFERMAGEM}

REGISTRO:

Data da consulta de enfermagem:

Endereço residencial:

Munićpio: Vassouras

1, Identificação

1.1 Sexo

( ) Masculino

( ) Feminino

1.2 Idade:

\subsection{Profissão/Atividade}

1.4 Local de trabalho/atividade

1.5 Escolaridade

( ) Analfabeto

( ) Alfabetizado

( ) $1^{\text {10 grau }}$

( ) $2 \%$ grau

( ) 3\% grau

( ) Completo

( ) Incompleto

2 Anmenese dirigida para controle: PA, Peso, Estatura e Pulso

2.1 Já aferiu anteriormente sua PA?

( ) Sim

2.1.1 Se afirmativo, qual $€$ a avaliação de sua PA?

( ) Não sabe

( ) Normal

( ) Anormal

( ) Especificar os valores:

2.2 Faz tratamento da hipertensão arterial?

( ) Sim

( ) Não

( ) Abandonou o tratamento

2.2.1 Se afirmativo, qual e o tratamento atual?

( ) Dieta hipocalórica (para emagrecer)

( ) Dieta hipossódica

( ) Com drogas. Especificar:

2.3 Faz uso de drogas hormonais?

( ) Corticosteroide

( ) Anticoncepcional

( ) Outros. Especificar: 
2.4 Considere a lista, a seguir, e identifique seus problemas de saude passados.

( ) Formigueiro

( ) Cefaleia

( ) Dor na nuca

( ) Tontura

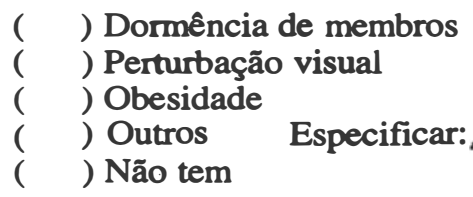

2.5 Considere a lista, a seguir, e identifique seus problemas de saúde atuais:

( ) Formigueiro

( ) Cefaleia

( ) Dor na nuca

( ) Tontura

( ) Dormência de membros
( ) Perturbação visual
( ) Obesidade
( ) Outros Especificar:
( Não tem

2.6 Tem familiar com problemas de PA?

) $\mathrm{Sim}$

( ) Não

Especificar o problema:

2.7 É tabagista?

) $\mathrm{Sim}$

) Não

Se afirmativo, especificar quantidade e freqüência:

2.8 Faz uso de bebidas alcoбlicas?

) $\mathrm{Sim}$

) Não

Especificar quantidade e freqüência:

2.9 Quais são seus hábitos alimentares?

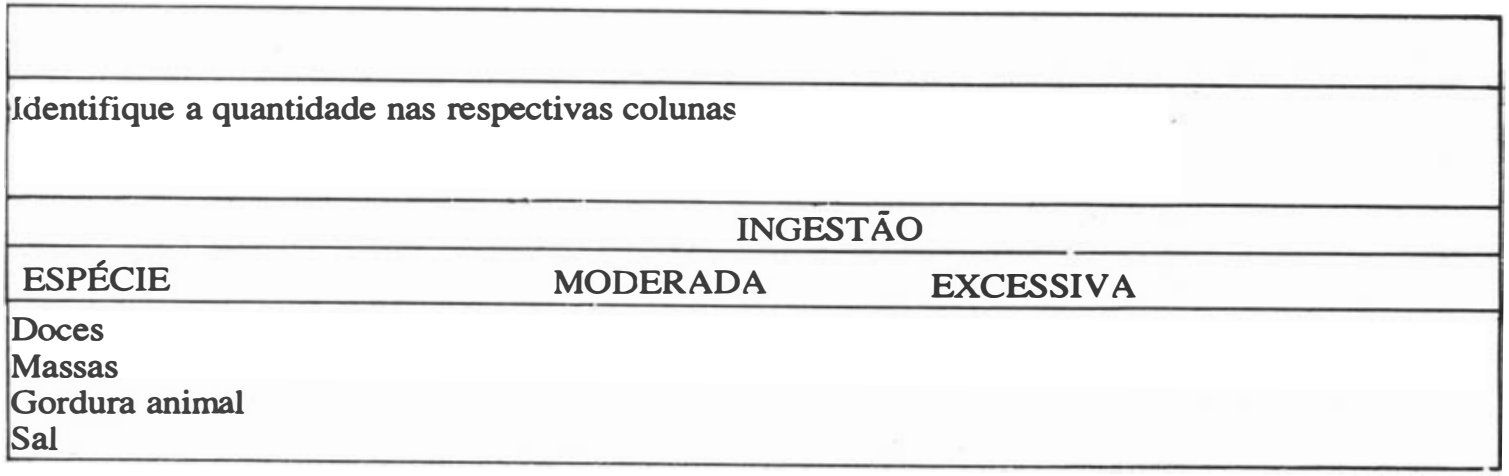

2.10 Indique suas condições de vida:

( ) Sedentarismo

( ) "Stress" no trabalho

( ) "Stress" na famnia

( ) Atividade física ativa. Especificar: 
2.11 Faz controle de peso

( ) Não

( ) Sim

( ) Especificar o valor de seu último peso verificado e data.

Peso:

Data:

3 Exame Clínico Especifico

3.1 Verificação

\begin{tabular}{|c|c|c|}
\hline BRAÇO DIREITO & BRAÇO ESQUERDO & PULSO \\
\hline Sistólica Diastólica & Sistólica Diastólica & \\
\hline & & \\
& & \\
\hline
\end{tabular}

\subsection{Aferições} antropométricas

Peso:

Estatura:

3.3 Outros dados de interesse clínico

4 Prescrição de Enfermagem

5 Agendamento de outras consultas de enfermagem e observações

6 Evolução do Controle da PA, Pulso e Peso nas consultas subsequentes

\begin{tabular}{ll|l}
\hline AFERIÇÕES PA PULSO PESO & OBSERVAÇÕES \\
\hline $1^{\mathrm{a}}$ & & \\
\hline $2^{\mathrm{a}}$ & & \\
\hline $3^{\mathrm{a}}$ & & \\
\hline
\end{tabular}


8. Encaminhamento:

ANEXO 2

CRONOGRAMA DE ATIVIDADES - 1991/1992

\begin{tabular}{l} 
Atividades \\
\hline $\begin{array}{l}\text { 1. Treinamento dos graduandos de enferma- } \\
\text { gem para a implementação da consulta }\end{array}$ \\
2. Divulgação da Consulta de Enfermagem en- \\
tre os grupos dá comunidade
\end{tabular}

3. Composição do Livro Saúde: sua forma de viver

4. Introdução da Consulta de Enfermagem

5. Palestra sobre Educação à saưde

6. Divulgação do Livro "Saúde: sua forma de viver" entre:

- Comunidade

- Instituições de Ensino

- Instituição de Saúde

- 60 Seminário Nacional de Pesquisa em Enfermagem

- 43 Congresso Brasileiro de Enfermagem

7. Reedição do Livro "Saúde: sua forma de viver"

- sem revisão

- revisão da obra

- ampliação

8. Implantação do Programa de Prevenção e Controle da Hipertensão Arterial junto aos Funcionários da FUSVE

- Consulta de Enfermagem

- Palestra sobre Educação à Saúde

Legenda:

$\square \begin{aligned} & \text { Realizada } \\ & \text { Planejada }\end{aligned}$

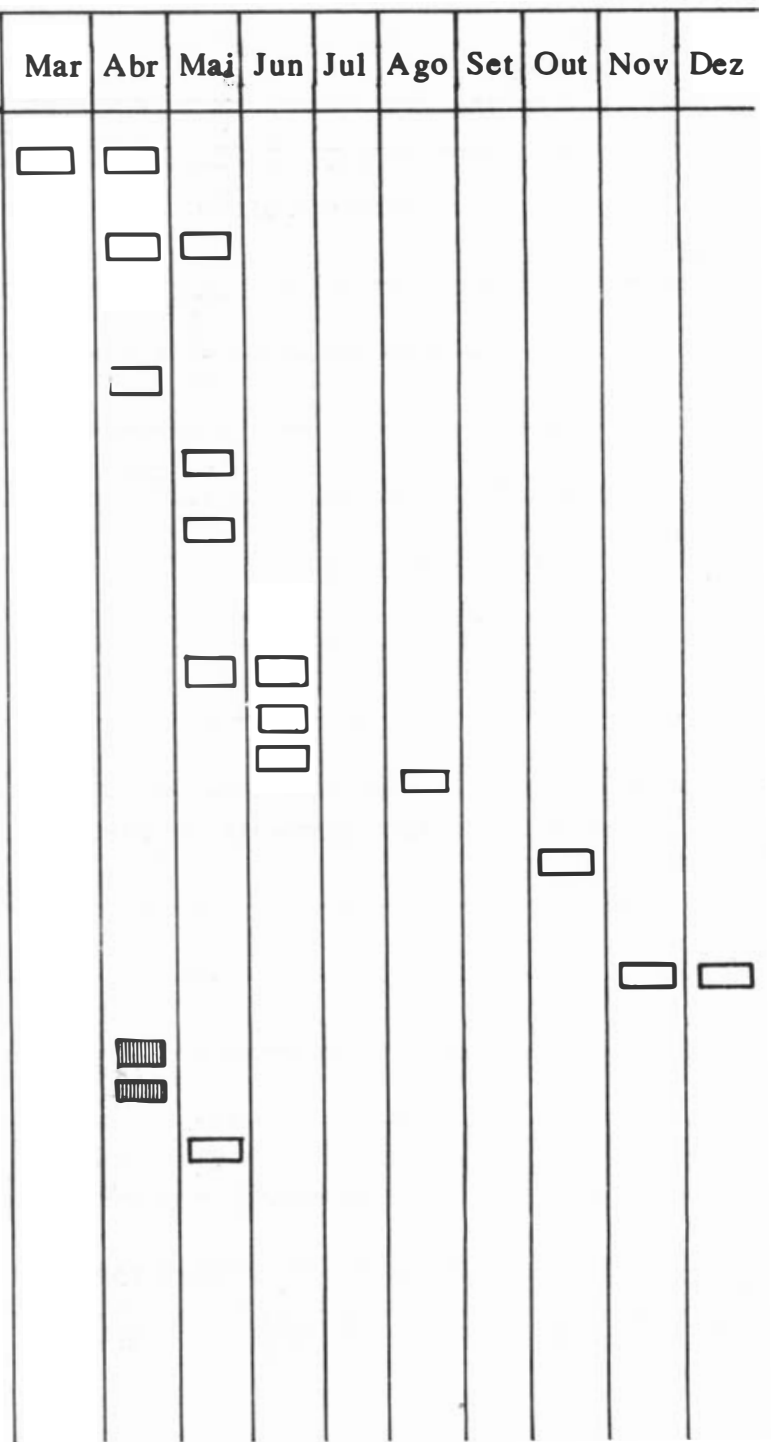

